\title{
Artéria radial versus veia safena para revascularização do miocárdio: metanálise (não houve diferença estatisticamente significante)
}

Radial artery versus saphenous vein to myocardial revascularization: meta-analysis (there is no statistically significant difference)

Rafael Fagionato LOCALI ${ }^{1}$, Enio BUFFOLO ${ }^{2}$, Roberto CATANI ${ }^{3}$

RBCCV 44205-827

Resumo

Objetivo: Comparar a efetividade do enxerto de artéria radial com o enxerto de veia safena para revascularização do miocárdio, em associação com a artéria torácica interna.

Método: Realizou-se uma revisão sistemática da literatura, utilizando uma estratégia de busca de artigos aplicada às bases de dados da MEDLINE e LILACS. Dois pesquisadores independentes realizaram a seleção dos artigos identificados, avaliando criteriosamente a metodologia dos artigos considerados relevantes para o tema. Somente os ensaios clínicos controlados e randomizados com adequado sistema de aleatorização foram incluídos. Em todas as situações em que ocorreu discordância entre os pesquisadores, foi realizada uma reunião de consenso. Não foi estipulada restrição quanto ao período pós-operatório para avaliação angiográfica do enxerto, o vaso tratado cirurgicamente e as características dos pacientes incluídos. Os resultados são expressos como Risco Relativo (RR), com 95\% de Intervalo de Confiança (CI), da comparação da efetividade entre a artéria radial e a veia safena.

Resultados: Com base nesses critérios foram incluídos três estudos. Não foi detectada diferença estatística entre a perviabilidade dos enxertos estudados (RR 0,53 [95\% IC 0,13 - 2,18]).

Conclusão: Apesar dos estudos serem de boa qualidade metodológica, não houve resultado estatisticamente significativo beneficiando um dos enxertos. O poder estatístico da metanálise é baixo. Portanto, são necessários novos ensaios clínicos controlados e randomizados, com tamanho de amostra adequado para detectar possíveis diferenças entre os tratamentos propostos.

Descritores: Artéria radial. Veia safena. Revascularização miocárdica. Metanálise.

1 - Graduando do curso de medicina.

2 - Professor Titular e Chefe da Disciplina de Cirurgia Cardiovascular da Universidade Federal de São Paulo - Escola Paulista de Medicina. 3 - Mestre. Professor da Disciplina de Cirurgia Cardiovascular da Universidade Federal de São Paulo - Escola Paulista de Medicina.

Trabalho realizado na Universidade Federal de São Paulo - Escola Paulista de Medicina (UNIFESP-EPM) Departamento de Cirurgia Disciplina de Cirurgia Cardiovascular, São Paulo, SP.

Endereço para correspondência:

Rafael Fagionato Locali. Rua Napoleão de Barros, 715, $3^{\circ}$ andar - Vila Clementino - São Paulo, SP - CEP: 04024-002.

E-mail: legolas@grad.unifesp.br

Artigo recebido em abril de 2006 Artigo aprovado em junho de 2006 


\section{Abstract}

Objective: To compare the effectiveness of radial artery graft with saphenous vein graft to myocardial revascularization, in association with the internal thoracic artery.

Methods: We made a systematic review of literature, using a strategy to search articles applied to MEDLINE and LILACS databases. Two independent researchers have carried through the election of identified articles, evaluating carefully the methodology of articles considered excellent for the subject. Only the randomized controlled trials, with adjusted randomization system have been enclosed. All the situations where the researchers didn't agree there was a consensus meeting. It hasn't been stipulated restriction for postoperative period to graft angiographic evaluation, the vessel treated and enclosed patient's characteristics. The results have been

\section{INTRODUÇÃO}

A doença arterial coronária é a principal causa de morte em praticamente todos os países do mundo, apesar dos avanços observados na cardiologia clínica e cirúrgica [1].

A revascularização do miocárdio (RM) é um procedimento estabelecido para tratar a doença arterial coronária avançada. Embora os resultados dessa operação sejam excelentes [2,3], benefícios tardios são influenciados pelo tipo de enxerto utilizado. Sabe-se que os enxertos de veia safena (VS), apesar de largamente empregados, possuem insatisfatória taxa de perviabilidade a longo prazo. Cerca de $15 \%$ dos enxertos de VS ocluem dentro do primeiro ano pós-operatório [4], e somente 50 a $60 \%$ dos enxertos estão patentes em dez anos de pós-operatório [5,6].

Dessa forma, o emprego da artéria torácica interna (ATI), seja esquerda, direita ou bilateral, como enxerto na RM é, sem dúvida, um dos mais importantes avanços no tratamento da doença coronariana dos últimos 25 anos. Essas artérias estão associadas com uma perviabilidade angiográfica superior, a longo prazo, quando comparada aos enxertos de VS, promovendo uma redução significativa da mortalidade [7-10]. Diante desses resultados, o emprego da ATI tornou-se padrão-ouro para RM, utilizando-se os enxertos de VS como complemento para alcançar a revascularização completa.

Por outro lado, o melhor resultado do enxerto arterial seduziu os cirurgiões a explorar outras artérias em complementação à ATI, visando à substituição dos enxertos de VS. Pesquisou-se, então, a artéria gastroepiplóica [1113], a artéria epigástrica inferior [14] e a artéria radial.

Dessas, a artéria radial (AR) é, atualmente, a mais utilizada. Inicialmente descrita em 1973, por Carpentier et al. [15], o uso da AR foi abandonado precocemente devido à sua propensão ao espasmo e à oclusão funcional $[16,17]$. expressed as Risk Relative (RR), with $95 \%$ of Confidence Interval (CI), to comparison the effectiveness between the radial artery and the saphenous vein.

Results: Based in these criteria three studies have been enclosed. We couldn't find statistic difference between grafts' patency studied (RR 0.53 [95\% IC $0.13-2.18]$ ).

Conclusion: Despite of the studies have good methodological quality, we have not observed a result significant statistically benefiting one of the grafts. The statistical power of the meta-analysis is low, therefore, it's necessary more randomized controlled trials, with adjusted sample size to detect possible differences between the considered treatments.

Descriptors: Radial artery. Saphenous vein. Myocardial revascularization. Meta-analysis.

Entretanto, com o advento de fármacos para prevenir o espasmo do enxerto e a adoção de melhores técnicas de dissecação, o interesse pela AR como um conduto arterial adicional foi revitalizado na década de 1990 [18].

Múltiplas instituições documentaram que a AR pode ser usada com mortalidade e morbidade aceitável e que os resultados angiográficos aproximavam-se dos da ATI [1927]. Por outro lado, a literatura possui dados limitados acerca dos resultados angiográficos tardios desses enxertos.

Uma recente série observacional, idealizada por Khot et al. [28], em 2004, mostrou redução da perviabilidade dos enxertos da AR quando comparados aos enxertos da ATI e da VS. Apesar de ser um estudo de baixo nível de significância, com alto potencial de vieses de seleção e seguimento, houve um abalo na credibilidade dos cirurgiões no emprego da AR na RM. Por isso, decidimos realizar uma revisão sistemática da literatura com metanálise para avaliar a efetividade do enxerto da AR na RM, comparando sua patência com o enxerto de VS, em associação ao enxerto da ATI.

\section{OBJETIVO}

Comparar a efetividade do enxerto da artéria radial ao da veia safena, em complementação ao enxerto da artéria torácica interna, para revascularização do miocárdio.

\section{MÉTODO}

\section{Critérios de inclusão de estudos na revisão}

\section{Tipos de estudos}

Ensaios clínicos controlados e randomizados ou quasirandomizados que comparavam a perviabilidade dos enxertos de AR e VS em associação ao enxerto da ATI. Estudos duplo-cegos não são possíveis nesse tipo de intervenção, entretanto, o mascaramento foi avaliado, mas 
não foi considerado como um requisito decisivo para inclusão ou exclusão dos estudos.

\section{Tipo de participantes}

Pacientes coronariopatas, independentemente da idade e da etnia, que necessitaram de uma intervenção cirúrgica para RM devido a obstrução superior a 70\% em qualquer vaso coronário. A necessidade de revascularização foi considerada a partir dos sinais e sintomas clínicos, laboratoriais, eletrocardiográficos e coronariográficos do paciente.

\section{Tipos de intervenções}

Foi avaliada a perviabilidade dos enxertos de AR e VS. Não foi considerado o número de pacientes, mas sim a quantidade de enxertos que cada um possuía. Os enxertos a comparados pertenciam ao mesmo paciente ou a pacientes distintos.

\section{Tipos de resultados medidos}

Oclusão do vaso 1 ano e 5 anos após o procedimento cirúrgico.

\section{Estratégia de busca para identificação dos estudos}

Foi realizada busca nas bases de dados eletrônicas da MEDLINE via PubMed e da LILACS via Biblioteca Virtual de Saúde até setembro de 2005. A estratégia de busca utilizada foi formulada a partir dos descritores e palavras relevantes para o tema da revisão, agrupados de forma a filtrar todos os artigos amplamente. Os autores optaram por uma estratégia com filtro de alta sensibilidade, e não foi feita restrição quanto à língua de publicação do artigo, diminuindo o viés de publicação.

\section{Estratégia da busca para MEDLINE}

(Aortocoronary Bypass OR Aortocoronary Bypasses OR Bypass, Aortocoronary OR Bypasses, Aortocoronary OR Bypass Surgery, Coronary Artery OR Coronary Artery Bypass Grafting OR Coronary Artery Bypass Surgery OR Bypass, Coronary Artery OR Artery Bypass, Coronary OR Artery Bypasses, Coronary OR Bypasses, Coronary Artery OR Coronary Artery Bypasses OR CABG OR Coronary artery bypass graft surgery OR CABG surgery OR Coronary Artery Bypass, Off Pump OR Beating Heart Coronary Artery Bypass OR Coronary Artery Bypass, Beating Heart OR OffPump Coronary Artery Bypass OR Off Pump Coronary Artery Bypass OR Off-pump coronary artery bypass graft surgery OR Off pump coronary artery bypass graft surgery OR Offpump coronary artery bypass grafting OR Off pump coronary artery bypass grafting OR OPCAB OR Off-pump CABG surgery OR Off pump CABG surgery) AND ((Arteries, Radial OR Artery, Radial OR Radial Arteries OR RA OR RA conduit* OR RA graft* OR Radial arterial conduit* OR Radial arterial graft* OR Radial aortocoronary bypass OR Radial aortocoronary bypass graft* OR Radial by pass OR Radial bypass OR Radial bypass graft* OR Radial bypass graft surgery OR Radial bypass operation OR Radial bypass surgery OR Radial conduit flap OR Radial conduits OR Radial coronary OR Radial coronary anastomoses OR Radial coronary bypass graft* OR Radial coronary bypass surgery) AND (Saphenous Veins OR Vein, Saphenous OR Veins, Saphenous OR SVG OR Saphenous allograft* OR Saphenous aortocoronary bypass OR Saphenous aortocoronary bypass graft* OR Saphenous by pass OR Saphenous bypass OR Saphenous bypass graft* OR Saphenous bypass graft surgery OR Saphenous bypass operation OR Saphenous bypass surgery OR Saphenous conduit flap OR Saphenous conduits OR Saphenous coronary OR Saphenous coronary anastomoses OR Saphenous coronary bypass graft* OR Saphenous coronary bypass surgery)) AND (randomized controlled trial[Publication Type] OR controlled clinical trial[Publication Type] OR randomized controlled trials[MeSH Terms] OR random allocation[MeSH Terms] OR double blind method[MeSH Terms] OR single blind method[MeSH Terms] OR clinical trial[Publication Type] OR clinical trials[MeSH Terms] OR (clinical*[Text Word] AND trial*[Text Word]) OR single*[Text Word] OR double*[Text Word] OR treble*[Text Word] OR triple*[Text Word] OR placebos[MeSH Terms] OR placebo*[Text Word] OR random*[Text Word] OR research design[MeSH Terms] OR comparative study[MeSH Terms] OR evaluation studies[MeSH Terms] OR follow-up studies[MeSH Terms] OR prospective studies[MeSH Terms] OR control*[Text Word] OR prospectiv*[Text Word] OR volunteer*[Text Word]).

\section{Estratégia de busca para LILACS}

(Aortocoronary Bypass OR Aortocoronary Bypasses OR Bypass, Aortocoronary OR Bypasses, Aortocoronary OR Bypass Surgery, Coronary Artery OR Coronary Artery Bypass Grafting OR Coronary Artery Bypass Surgery OR Bypass, Coronary Artery OR Artery Bypass, Coronary OR Artery Bypasses, Coronary OR Bypasses, Coronary Artery OR Coronary Artery Bypasses OR CABG OR Coronary artery bypass graft surgery OR CABG surgery OR Coronary Artery Bypass, Off Pump OR Beating Heart Coronary Artery Bypass OR Coronary Artery Bypass, Beating Heart OR OffPump Coronary Artery Bypass OR Off Pump Coronary Artery Bypass OR Off-pump coronary artery bypass graft surgery OR Off pump coronary artery bypass graft surgery OR Offpump coronary artery bypass grafting OR Off pump coronary artery bypass grafting OR OPCAB OR Off-pump CABG surgery OR Off pump CABG surgery) AND (Arteries, Radial OR Artery, Radial OR Radial Arteries OR RA OR RA conduit\$ OR RA graft\$ OR Radial arterial conduit\$ OR Radial arterial graft\$ OR Radial aortocoronary bypass OR Radial 
aortocoronary bypass graft\$ OR Radial by pass OR Radial bypass OR Radial bypass graft\$ OR Radial bypass graft surgery OR Radial bypass operation OR Radial bypass surgery OR Radial conduit flap OR Radial conduits OR Radial coronary OR Radial coronary anastomoses OR Radial coronary bypass graft\$ OR Radial coronary bypass surgery) AND (Saphenous Veins OR Vein, Saphenous OR Veins, Saphenous OR SVG OR Saphenous allograft\$ OR Saphenous aortocoronary bypass OR Saphenous aortocoronary bypass graft\$ OR Saphenous by pass OR Saphenous bypass OR Saphenous bypass graft\$ OR Saphenous bypass graft surgery OR Saphenous bypass operation OR Saphenous bypass surgery OR Saphenous conduit flap OR Saphenous conduits OR Saphenous coronary OR Saphenous coronary anastomoses OR Saphenous coronary bypass graft\$ OR Saphenous coronary bypass surgery) AND ((Pt randomized controlled trial) OR (Pt controlled clinical trial) OR (Mh randomized controlled trials) OR (Mh random allocation) OR (Mh double blind method) OR (Mh single blind method) AND NOT (Ct animal) AND NOT (Ct human and Ct animal) OR (Pt clinical trial) OR (Ex E05.318.760.535\$) OR (Tw clin\$) AND (Tw trial\$) OR (Tw ensa) OR (Tw estud\$) OR (Tw experim\$) OR (Tw investiga\$) OR (Tw singl\$) OR (Tw simple\$) OR (Tw doubl\$) OR (Tw doble\$) OR (Tw duplo\$) OR (Tw trebl\$) OR (Tw trip\$) AND (Tw blind\$) OR (Tw cego\$) OR (Tw ciego\$) OR (Tw mask\$) OR (Tw mascar\$) OR (Mh placebos) OR (Tw placebo\$) OR (Tw random\$) OR (Tw randon\$) OR (Tw casual\$) OR (Tw acaso\$) OR (Tw azar) OR (Tw aleator\$) OR (Mh research design) AND NOT (Ct animal) AND NOT (Ct human and Ct animal) OR (Ct comparative study) OR (Ex E05.337\$) OR (Mh follow-up studies) OR (Mh prospective studies) OR (Tw control\$) OR (Tw prospectiv\$) OR (Tw volunt\$) OR (Tw volunteer\$) AND NOT ((Ct animal) AND NOT (Ct human and Ct animal))).

\section{Método padronizado de revisão}

\section{Seleção dos estudos}

Dois pesquisadores independentes selecionaram os ensaios por inclusão ou exclusão, depois de analisar os títulos e resumos dos estudos identificados, usando a estratégia de busca. Os ensaios selecionados foram comparados e qualquer discrepância foi resolvida por discussão e consenso. A seguir, os ensaios incluídos foram avaliados, na íntegra, pelos dois pesquisadores, independentemente, para assegurar que estes obedeciam aos critérios de inclusão estipulados. Finalmente, os artigos selecionados para a revisão foram confirmados para evitar inclusão de dados publicados em duplicata.

\section{Qualidade de avaliação}

Dois pesquisadores, independentemente, avaliaram a qualidade metodológica dos estudos incluídos. Verificou- se a adequação da alocação dos pacientes, estipulando uma classificação dos estudos em categorias, nas quais a categoria A (sigilo de alocação adequado e descrito na metodologia do estudo), B (sigilo de alocação mencionado e não descrito na metodologia do estudo), C (sigilo de alocação inadequado) e D (estudo com alocação não aleatória). Qualquer diferença de opinião foi resolvida por discussão e consenso.

\section{Extração dos dados}

Os dados dos estudos foram extraídos por dois pesquisadores independentemente, usando um formulário padronizado. Os formulários foram montados com base nos dados previamente definidos como relevantes para o tema. Quando necessário, os autores dos estudos primários foram contatados para adição de informação ou dados nos resultados de seus estudos.

\section{Qualidade metodológica dos estudos incluídos}

Todos os estudos incluídos foram randomizados ou quasi-randomizados, isto é, somente foram incluídos os estudos classificados nas categorias A, B e C. Os estudos de categoria $\mathrm{D}$ foram excluídos desta Revisão Sistemática. Dois estudos foram classificados na categoria A (Dessai et al., 2004; Buxton et al., 2003) [29,30] e um estudo foi classificado como categoria B (Muneretto et al., 2004) [31].

\section{RESULTADOS}

Após realizar a extração de todos os dados relevantes dos estudos, avaliando sua qualidade, estes foram plotados no programa Review Manager - RevMan 4.2.8 - para análise. Comparações dos efeitos estimados entre as duas intervenções de interesse foram expressas como Risco Relativo (RR), com 95\% de Intervalo de Confiança (IC).

Foram selecionados 398 artigos nas bases de dados da MEDLINE e LILACS. Destes, apenas três artigos correspondiam aos critérios de inclusão do tipo de estudo, ou seja, eram ensaios clínicos controlados e randomizados ou quasi-randomizados.

A metanálise dos estudos, com 1116 enxertos estudados até a data da finalização dessa revisão sistemática, é apresentada como análise de subgrupos, em função da avaliação da patência dos enxertos em doze meses e em cinco anos. Os resultados da metanálise dos subgrupos e global estão apresentados em modelo randômico (Figura 1).

Observa-se que para patência de 12 meses não existe diferença estatística entre os grupos (RR 0,31 [95\% IC 0,05 1,84]), assim como para patência em cinco anos (RR 2,75 [95\% IC 0,31 - 24,52]). A estimativa do efeito global não mostra proporções de eventos estatisticamente significantes entre ambos os grupos de comparação, quando expressos como risco relativo (RR 0,53 [95\% IC 0,13 - 2,18]). 


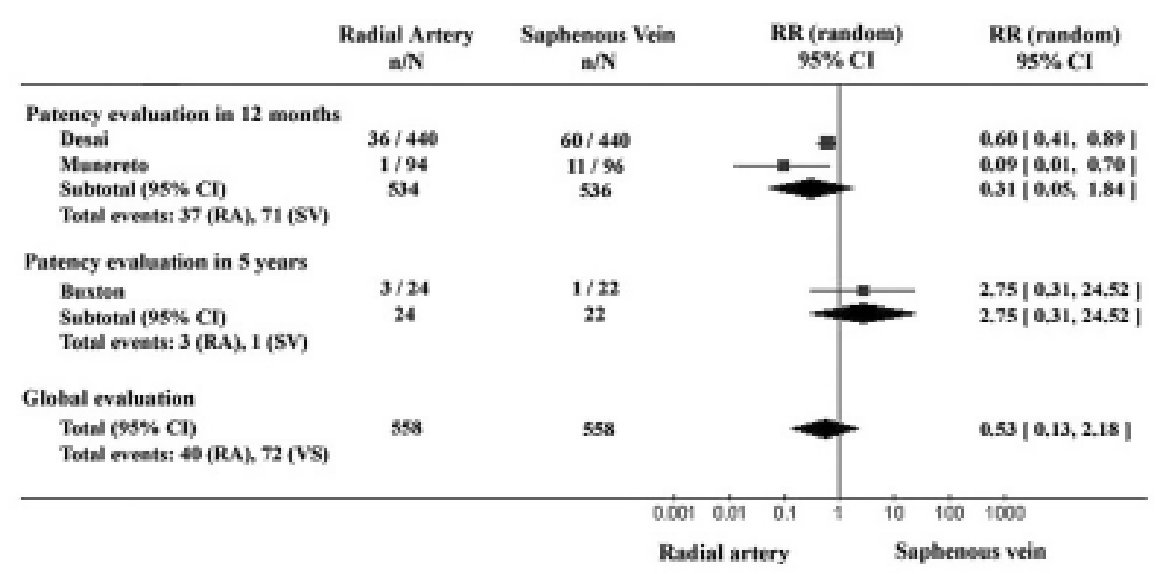

Fig. 1 - Metanálise dos subgrupos e global. Para a patência de 12 meses não existe diferença estatística entre os grupos, assim como para patência em cinco anos. A estimativa do efeito global não mostra proporções de eventos estatisticamente significantes entre os grupos de comparação quando expressos como risco relativo

\section{DISCUSSÃO}

A cirurgia de RM é, sem dúvida, o tratamento de escolha diante de pacientes com grave doença coronária. Esse procedimento requer enxertos vasculares, tanto venosos quanto arteriais, com a finalidade de otimizar a irrigação sangüínea do tecido miocárdico das regiões pós-estenóticas. A partir disso, uma série de estudos foram realizados, a fim de comparar a eficácia dos enxertos venosos e arteriais. Comprovou-se que a viabilidade dos enxertos da ATI esquerda é superior à dos enxertos da VS [7,9,10], os quais, atualmente, são utilizados como complemento dos enxertos da ATI para alcançar a RM completa. Com isso, os cirurgiões buscaram por novas artérias que poderiam substituir os enxertos de VS. Atualmente, a artéria radial é a mais utilizada e estudada para esse procedimento, em associação à ATI.

Sabe-se que a patência do enxerto, independentemente se arterial ou venoso, depende tanto de fatores inerentes ao paciente, como a presença de comorbidades (diabetes, dislipidemia, hipertensão arterial e insuficiência renal), quanto de fatores relacionados à metodologia de dissecação, à preservação e à proteção farmacológica do vaso e técnica cirúrgica de implante [10]. Além disso, o território do vaso coronário alvo do tratamento também está relacionado a uma maior ou menor patência do enxerto [21].

Uma série de estudos observacionais indicou que a patência dos enxertos da AR é superior aos da VS [32,33]. Por outro lado, Khot et al., em 2004, concluíram que a patência dos enxertos de AR é inferior à patência de todos os outros vasos utilizados para RM [28]. No entanto, todos esses estudos são não-randomizados, não-controlados e observacionais, seja prospectivos ou retrospectivos, sendo, portanto, de mínima confiabilidade e sujeitos a considerável quantidade de vieses. Nesse sentido, realizamos uma revisão sistemática da literatura para avaliar a patência dos enxertos de AR quando comparados aos da VS.

Traçamos um objetivo amplo, não restringindo o período pós-operatório para avaliação angiográfica do enxerto, o vaso tratado cirurgicamente e as características dos pacientes incluídos. Essa conduta foi adotada para contrapor a extrema carência de ensaios clínicos controlados e randomizados que abrangem esse tema. Foram incluídos três ensaios randomizados nesta revisão sistemática. As bases de dados revisadas detêm indexação de considerável parcela dos periódicos de maior renome na esfera da cirurgia cardiovascular, evidenciando que houve insignificante viés de publicação. A estratégia de busca utilizada possui alta sensibilidade, abordando todos os estudos relacionados ao tema. Os ensaios clínicos controlados e randomizados ou quasirandomizados foram eleitos para inclusão nesta revisão sistemática por possuírem a maior confiabilidade e nível de evidência, com menor erro de tipo $1(\alpha)$ e tipo $2(\beta)$. O mascaramento não foi exigido para inclusão, e foi avaliado o número de enxertos patentes e não o número de pacientes.

O ensaio clínico controlado randomizado proposto por Dessai et al. [29], publicado em 2004, compara a patência, em 12 meses, do enxerto de AR ao de VS. Utiliza metodologia reprodutível e científica, com adequado método de randomização, e quantidade razoável de pacientes $(n=440)$. As avaliações angiográficas foram pré-determinadas e, em seus resultados, observa-se melhor patência do enxerto de AR do que da VS.

Muneretto et al. [30], também em publicação de 2004, compararam a patência dos enxertos de AR e VS em 12 meses. Foram avaliados os resultados angiográficos de 152 pacientes, os quais foram distribuídos em dois grupos. O número de participantes é relativamente pequeno, aumentando a incerteza dos resultados e conferindo um intervalo de confiança considerável. A metodologia é reprodutível, com as angiografias programadas previamente, entretanto, a forma de randomização dos pacientes não foi descrita. Concluem afirmando que o enxerto da AR tem melhor patência do que os da VS.

Outro ensaio clínico controlado randomizado foi idealizado por Buxton et al. [31], em 2003. Estes autores recrutaram 438 pacientes, que foram randomizados em dois grupos. O primeiro deles, com 285 pacientes, que foram randomizados em outros dois grupos, um com 140 pacientes que receberam enxertos de AR e outro com 145 pacientes que receberam enxertos da ATI direita. O segundo grupo 
continha 153 pacientes, que foram randomicamente distribuídos em outros dois grupos, um com 73 pacientes que receberam enxertos da $\mathrm{AR}$ e outro com 80 pacientes que receberam enxerto da VS. A metodologia é adequada, com programação de avaliação angiográfica de $40 \%$ dos pacientes em cinco anos e $60 \%$ dos pacientes em dez anos. O número de pacientes não foi adequado, e os resultados de cinco anos não apontavam diferença estatística entre os grupos com enxerto de artéria radial e veia safena. Os dados da patência em dez anos não estão disponíveis ainda.

É digno de nota que todos esses ensaios clínicos possuíam em sua metodologia um estudo angiográfico pré-determinado, mesmo com o paciente não apresentando sintomas isquêmicos. Estudos angiográficos baseados em sintomas não oferecem uma representatividade satisfatória da população, pois é esperado que a taxa de falência dos enxertos nesses pacientes seja maior [10]. Buxton et al. [10], em 2005, publicaram um estudo cujo resultado expressava que a patência dos enxertos da ATI, AR e VS era maior nos protocolos em que os pacientes eram avaliados independentes dos sintomas do que naqueles em que o paciente era submetido à exame angiográfico mediante os sintomas.

Nos resultados, apresentamos um gráfico de efeito global, editado em modelo randômico. Este modelo valoriza as heterogeneidades clínicas e metodológicas presentes nos estudos, relevando-as nos cálculos estatísticos. Portanto, espera-se que o resultado seja mais confiável, evidenciando melhor as possíveis heterogeneidades já mencionadas, visto que os estudos incluídos não eram homogêneos. A metodologia de seleção de pacientes, os critérios diagnósticos, as formas de avaliação angiográfica e os procedimentos cirúrgicos não eram semelhantes entre os ensaios clínicos incluídos. Nesse sentido, não foi observada qualquer diferença entre os efeitos do uso do enxerto de AR e VS.

Entretanto, apesar dos estudos incluídos possuírem boa qualidade, com valores satisfatórios na escala de qualidade de estudos de Jadad et al. [34], o poder estatístico da metanálise é baixo, pois o número de enxertos acumulados nesta revisão foi muito pequeno $(\mathrm{n}=1116)$. Apesar disso, existe uma maior proporção de patência do enxerto de artéria radial quando comparado com o enxerto de veia safena, expressos nos resultados de modelo randômico. Porém, uma afirmação plena e convicta de que o enxerto radial é melhor do que o enxerto safeno necessita de que mais ensaios clínicos controlados e randomizados sejam realizados, agrupando uma casuística expressiva, seguindo um padrão metodológico uniforme e homogêneo. Os autores sugerem que o desenho metodológico de novos ensaios clínicos seja embasado pela publicação de Fremes [35], 2000. Esta metodologia, com certas adaptações pode ser utilizada para condução de uma série de ensaios clínicos controlados e randomizados.

\section{REFERÊNCIAS}

1. National Center for Health. Statistics and the Commission of Professional Hospital Activities. Washington DC;1992.

2. Kirklin JW, Naftel DC, Blackstone EH, Pohost GM. Summary of a consensus concerning death and ischemic events after coronary artery bypass grafting. Circulation. 1989;79(6 pt 2):I81-91.

3. American College of Cardiology/American Heart Association Task Force on Assessment of Diagnostic and Therapeutic Cardiovascular Procedures (Subcommittee on Coronary Artery Bypass Graft Surgery). Guidelines and indications for coronary artery bypass graft surgery. J Am Coll Cardiol. 1991;17(3):543-89.

4. Fremes SE, Levinton C, Naylor CD, Chen E, Christakis GT, Goldman BS. Optimal antithrombotic therapy following aortocoronary bypass: a meta-analysis. Eur J Cardiothorac Surg. 1993;7(4):169-80.

5. Bourassa MG, Fisher LD, Campeau L, Gillespie MJ, McConney M, Lesperance J. Long-term fate of bypass grafts: the Coronary Artery Surgery Study (CASS) and Montreal Heart Institute experiences. Circulation. 1985;72(6 pt 2):V71-8.

6. Kouchoukos NT, Karp RB, Oberman A, Russell RO Jr, Alison HW, Holt JH Jr. Long-term patency of saphenous veins for coronary bypass grafting. Circulation. 1977;56(Suppl 3):189.

7. Loop FD, Lytle BW, Cosgrove DM, Stewart RW, Goormastic $\mathrm{M}$, Williams GW et al. Influence of the internal mammary artery graft on 10-year survival and other cardiac events. N Engl J Med. 1986;314(1):1-6.

8. Cameron A, Davis KB, Green G, Schaff HV. Coronary bypass surgery with internal-thoracic-artery grafts-effects on survival over a 15-year period. N Engl J Med. 1996;334(4):216-9.

9. Lytle BW, Loop FD, Cosgrove DM, Ratliff NB, Easley K, Taylor PC. Long-term (5 to 12 years) serial studies of internal mammary artery and saphenous vein coronary bypass grafts. J Thorac Cardiovasc Surg. 1985;89(2):248-58.

10. Buxton BF, Durairaj M, Hare DL, Gordon I, Moten S, Orford V et al. Do angiographic results from symptom-directed studies reflect true graft patency? Ann Thorac Surg. 2005;80(3):896-901

11. Buffolo E, Maluf M, Barone B, Andrade JC, Gallucci C. Direct myocardial revascularization with the left gastro-epiploic artery. A new alternative to aortocoronary bypass. A case report. Arq Bras Cardiol. 1987;48(3):167-71.

12. Suma H, Takeuchi A, Hirota Y. Myocardial revascularization with combined arterial grafts utilizing the internal mammary and the gastroepiploic arteries. Ann Thorac Surg. 1989;47(5):712-5. 
13. Bergsma TM, Grandjean JG, Voors AA, Boonstra PW, den Heyer P, Ebels T et al. Low recurrence of angina pectoris after coronary artery bypass graft surgery with bilateral internal thoracic and right gastroepiploic arteries. Circulation. 1998;97(24):2402-5.

14. Puig LB, Ciongolli W, Cividanes GV, Dontos A, Kope L, Bittencourt D et al. Inferior epigastric artery as a free graft for myocardial revascularization. J Thorac Cardiovasc Surg. 1990;99(2):251-5.

15. Carpentier A, Guermonprez JL, Deloche A, Frechette C, DuBost C.The aorta-to-coronary radial artery bypass graft: a technique avoiding pathological changes in grafts. Ann Thorac Surg. 1973;16(2):111-21.

16. Curtis JJ, Stoney WS, Alford WC Jr, Burrus GR, Thomas CS Jr. Intimal hyperplasia: a cause of radial artery aortocoronary bypass graft failure. Ann Thorac Surg. 1975;20(6):628-35.

17. Chiu CJ. Why do radial artery grafts for aortocoronary bypass fail? A reappraisal. Ann Thorac Surg. 1976;22(6):520-3.

18. Acar C, Jebara VA, Portoghese M, Viesen B, Pagny JY, Grare $P$ et al. Revival of the radial artery for coronary artery bypass grafting. Ann Thorac Surg. 1992;54(4):652-60.

19. Weinschelbaum EE, Macchia A, Caramutti VM, Machain HA, Raffaelli HA, Favaloro MR et al. Myocardial revascularization with radial and mammary arteries: initial and mid-term results. Ann Thorac Surg. 2000;70(4):1378-83.

20. Amano A, Hirose H, Takahashi A, Nagano N. Coronary artery bypass grafting using the radial artery: midterm results in a Japanese institute. Ann Thorac Surg. 2001;72(1):120-5.

21. Tatoulis J, Royse AG, Buxton BF, Fuller JA, Skillington PD, Goldblatt JC et al. The radial artery in coronary surgery: a 5year experience. Clinical and angiographic results. Ann Thorac Surg. 2002;73(1):143-8.

22. Costa FD, Costa IA, Poffo R, Abuchaim D, Gaspar R, Garcia $\mathrm{L}$ et al. Myocardial revascularization with the radial artery: a clinical and angiographic study. Ann Thorac Surg. 1996;62(2):475-80.

23. Dallan LA, Oliveira SA, Lisboa LA, Platania F, Jatene FB, Iglesias JCR et al. Revascularização completa do miocárdio com uso exclusivo de enxertos arteriais. Rev Bras Cir Cardiovasc 1998;13(3):187-93

24. Fisk RL, Brooks CH, Callaghan JC, Dvorkin J. Experience with the radial artery graft for coronary artery bypass. Ann Thorac Surg. 1976;21(6):513-8.

25. He GW. Arterial grafts for coronary surgery: vasospasm and patency rate. J Thorac Cardiovasc Surg. 2001;121(3):431-3.

26. Parolari A, Rubini P, Alamanni F, Cannata A, Xin W, Gherli T et al. The radial artery: which place in coronary operation? Ann Thorac Surg. 2000;69(4):1288-94.

27. Possati G, Gaudino M, Alessandrini F, Luciani N, Glieca F, Trani $\mathrm{C}$ et al. Midterm clinical and angiographic results of radial artery grafts used to myocardial revascularization. J Thorac Cardiovasc Surg. 1998;1166):1015-21.

28. Khot UN, Friedman DT, Pettersson G, Smedira NG, Li J, Ellis SG. Radial artery bypass grafts have an increased occurrence of angiographically severe stenosis and occlusion compared with left internal mammary arteries and saphenous vein grafts. Circulation. 2004;109(17):2086-91.

29. Desai ND, Cohen EA, Naylor CD, Fremes SE. Randomized comparison of radial-artery and saphenous-vein coronary bypass grafts. N Engl J Med. 2004;351(22):2302-9.

30. Muneretto C, Bisleri G, Negri A, Manfredi J, Carone E, Morgan JA et al. Left internal thoracic artery-radial artery composite grafts as the technique of choice for myocardial revascularization in elderly patients: a prospective randomized evaluation. J Thorac Cardiovasc Surg. 2004;127(1):179-84

31. Buxton BF, Raman JS, Ruengsakulrach P, Gordon I, Rosalion A, Bellomo R et al. Radial artery patency and clinical outcomes: five-year interim results of a randomized trial. J Thorac Cardiovasc Surg. 2003;125(6):1363-71.

32. Possati G, Gaudino M, Prati F, Alessandrini F, Trani C, Glieca F. Long-term results of the radial artery used for myocardial revascularization. Circulation. 2003;108(11):1350-4.

33. Modine T, Al-Ruzzeh S, Mazrani W, Azeem F, Bustami M Ilsley $\mathrm{C}$ et al. Use of radial artery graft reduces the morbidity of coronary artery bypass graft surgery in patients aged 65 years and older. Ann Thorac Surg. 2002;74(4):1144-7.

34. Jadad AR, Moore RA, Carroll D, Jenkinson C, Reynolds DJ, Gavaghan DJ et al. Assessing the quality of reports of randomized clinical trials: is blinding necessary? Control Clin Trials. 1996;17(1):1-12.

35. Fremes SE. Multicenter Radial Artery Patency Study (RAPS): study design. Control Clin Trials. 2000;21(4):397-413. 